\title{
A Lagrangian model for wave-induced harbour oscillations
}

\author{
By J. A. ZELT AND F. RAICHLEN \\ W. M. Keck Laboratory of Hydraulics and Water Resources, Division of Engineering and \\ Applied Science, California Institute of Technology, Pasadena, CA 91125, USA
}

(Received 30 May 1989 and in revised form 24 August 1989)

\begin{abstract}
A set of equations in the Lagrangian description are derived for the propagation of long gravity waves in two horizontal directions for the purpose of determining the response of harbours with sloping boundaries to long waves. The equations include terms to account for weakly nonlinear and dispersive processes. A finite element formulation for these equations is developed which treats the propagation of transient waves in regions of arbitrary shape with vertical or sloping boundaries. Open boundaries are treated by specifying the wave elevation along the boundary or by applying a radiation boundary condition to absorb the waves leaving the computational domain. Nonlinear aspects of the interaction of long gravity waves with sloping boundaries and frequency dispersion due to non-hydrostatic effects are investigated. Results from the model are then compared with laboratory experiments of the response to long-wave excitation of a narrow rectangular harbour with a depth that decreases linearly from the entrance to the shore line.
\end{abstract}

\section{Introduction}

The amplitude of a wave within a harbour may be greatly amplified if the wave contains significant energy in a frequency band near one of its natural resonant modes of oscillation. For simple harbour geometries and depth variations the resonant modes can be predicted theoretically (see Zelt (1986) for a compilation of resonance characteristics of several types of narrow harbours), but for more complicated geometries, for transient excitation, and for cases where nonlinear effects are important, the harbour response can be determined only from experiments conducted with laboratory or numerical models. The reader is referred to Raichlen (1966, 1976), Wilson (1972), Miles (1974), Lepelletier (1980), and Zelt (1986) for reviews of this subject.

Virtually all numerical work related to the wave induced oscillations of harbours and bays has treated coastlines and harbour boundaries as vertical walls, i.e. by introducing an imaginary fixed vertical barrier near the shore, along which the water depth is always non-zero. In such cases the run-up of the waves must be determined by extrapolation, which can be inaccurate and generally does not conserve mass or momentum (Lynch \& Gray 1978). Also, the assumed boundary shape itself may change the response of the harbour or coastline by altering its resonant characteristics. To avoid this problem, techniques are required which can treat a zero water depth at the shore line; approaches which use the vertical wall approximation will not be discussed further here. More sophisticated methods attempt to conserve 
mass and momentum by explicitly computing both the vertical and the horizontal motion of the shore line, but the run-up of surface gravity waves on a sloping boundary is a very complicated process to treat numerically. Even if wave breaking does not occur, a nonlinear analysis is still complicated because the motion of the shore line represents a moving boundary which must be computed as part of the solution. Most treatments of sloping boundaries can be divided roughly into Eulerian or Lagrangian methods, although particular models can also combine aspects of both of these approaches.

Models cast in the Eulerian frame of reference can employ either fixed or deforming computational grids. Early work by Keller \& Keller $(1964,1965)$ and Sielecki \& Wurtele (1970) employed a fixed grid, although the shore line position was tracked between grid points. Gopalakrishnan \& Tung (1983) developed a finite element model for one horizontal dimension which used a fixed grid, except at the coastline where an element was allowed to deform to follow the shore line and to split into two elements if it became too stretched. Other investigators, such as Reid \& Bodine (1968), Houston \& Butler (1979), Yeh \& Chou (1979), and Tanaka, Ono \& Ishise (1980) simulated the moving shore line with a fixed grid by turning entire cells on and off at the boundary. Lynch \& Gray $(1978,1980)$ described a finite element technique to treat moving boundaries by using time dependent basis functions which were chosen so that the finite element grid deformed to track a moving shore line. This technique is appealing since it avoids the impulsive flooding or draining of entire cells and the need to track the shore line between grid points, although the introduction of deforming elements significantly increases the computational requirements.

Models cast in the Lagrangian frame of reference use fixed computational grids almost exclusively; that is the motivation for performing the computations in this frame of reference. Since the independent variables are the initial coordinates of the fluid particles, the computational grid does not distort, even as the shore line moves, although computational difficulties can result from excessive shearing motions which distort the grid in physical space. The linear problem was treated by Shuto (1967, 1968, 1972) and Shuto \& Goto (1978). However, if finite amplitude effects are neglected, Lagrangian models are equivalent to Eulerian models since all variables are referenced with respect to fixed positions in space. Therefore, only nonlinear models will be considered here. Heitner (1969, 1970), Goto (1979), and Pedersen \& Gjevik (1983) each developed nonlinear run-up models for wave propagation in one horizontal dimension, and Goto \& Shuto (1980) developed a finite difference model which treats two horizontal dimensions.

This paper describes a finite element technique to study the propagation of long waves in two horizontal dimensions in regions of arbitrary shape with vertical or sloping boundaries. A restriction is imposed that perfect reflection occurs at these boundaries, i.e. wave breaking is not modelled. Therefore, the work described in this paper is most applicable to studies involving waves which are long compared to the characteristic horizontal scale of the slope. A Lagrangian approach was chosen to simplify the treatment of the moving shore line. The equations of motion are of the Boussinesq type, i.e. both nonlinear and dispersive effects are included.

It is well known that in two or more dimensions pure Lagrangian techniques are susceptible to large shearing motions which distort the connectivity between fluid particles. Eulerian techniques with deforming computational grids are also susceptible to this problem, although it is possible to choose the motion of the lattice points appropriately to minimize this shear (Lynch \& Gray 1980); however, this 
process can be slow and involved. Currents responsible for such shear can be generated by periodic disturbances (although mean currents are not essential for generating large distortions). For example, a longshore current can be generated in the surf zone by wave breaking if the incident waves approach the shore at an oblique angle (Longuet-Higgins 1970). Mean currents perpendicular to the coast can be produced by periodic longshore variations in the set-up (associated with wave breaking) caused by synchronous and subharmonic edge waves generated through a weak resonant interaction with the incident wave field (Bowen 1969; Bowen \& Inman 1969; Guza \& Davis 1974). However, since wave breaking is not simulated in the model, these sources of mean current shear do not apply here and a Lagrangian approach is feasible.

In $\S 2$ the long-wave equations in the Lagrangian description are developed and a finite element formulation is described to solve these equations numerically to determine the response of arbitrarily shaped harbours or bays with sloping boundaries to incident long waves. The treatment of sloping boundaries along the periphery of a harbour is investigated. The treatment of frequency dispersion due to non-hydrostatic effects is investigated by comparing results with known theoretical dispersion relations. In $\$ 3$ harbour response laboratory experiments are described. The finite element model is applied to a long narrow harbour whose depth decreases linearly from its entrance to the shore line, and the response of the harbour to long waves obtained numerically is compared to the laboratory experiments. Finally, in $\S 4$ the results are summarized and conclusions are drawn.

\section{Theory}

In this section the long-wave equations in the Lagrangian coordinate system will be developed first, followed by a description of a general finite element formulation of these equations. Finally, this formulation will be specialized to treat the long-wave excitations of harbours and bays; several aspects of this treatment which are related to non-hydrostatic and nonlinear effects of wave propagation up sloping boundaries will be addressed.

\subsection{Long-wave equations in the Lagrangian description}

The long-wave equations in the Lagrangian description for wave propagation in two horizontal dimensions will be obtained by depth integrating the three-dimensional equations of motion. All dependent variables will be expressed in the Lagrangian description. The variables $(x, y, z)$ denote Cartesian coordinates with the $z$-axis oriented vertically upwards, referenced from the still water surface. These coordinates also define the position of a water particle which at time $t=0$ was located at the reference location $(a, b, c)$. The pressure is given by $p$; its reference value is the quantity $-\rho g c$, i.e. the hydrostatic pressure below the still water surface at the initial location of a fluid particle.

The Lagrangian equations for fluid motion in three dimensions can be obtained from either a variational approach (Eckart 1963) or a control volume approach (Lamb 1932). Under the assumption that the fluid density, $\rho$, is constant, the mass conservation equation can be stated simply as (Lamb 1932):

$$
J \equiv \frac{\partial(x, y, z)}{\partial(a, b, c)}=1
$$


Here $J$ is the Jacobian of the transformation between the $(x, y, z)$ and the $(a, b, c)$ variables. Similarly, if the viscous terms are neglected, the momentum equations can be expressed as

$$
\frac{\partial^{2} x}{\partial t^{2}}=-\frac{1}{\rho} \frac{\partial(p, y, z)}{\partial(a, b, c)}, \quad \frac{\partial^{2} y}{\partial t^{2}}=-\frac{1}{\rho} \frac{\partial(x, p, z)}{\partial(a, b, c)}, \quad \frac{\partial^{2} z}{\partial t^{2}}+g=-\frac{1}{\rho} \frac{\partial(x, y, p)}{\partial(a, b, c)},
$$

where use has been made of (1). Assuming all motion starts from rest at time $t=0$, with the free surface described by $c=0$, these equations must be solved subject to the boundary conditions

$$
p(a, b, 0)=0, \quad z(a, b,-h(a, b))=-h(x, y) .
$$

The first expression is a dynamic boundary condition which states that the pressure is zero at the free surface, and the second is a kinematic boundary condition which states that particles which lie on the bottom at time $t=0$, i.e. particles for which $c=-h(a, b)$, remain on the bottom thereafter. Notice that there is only one boundary condition to impose at the free surface. In the Eulerian description there are two boundary conditions because the position of the free surface is an extra unknown to be determined as part of the problem. Since the free surface is a material surface, its position is fixed in the Lagrangian description and an extra variable is not needed to keep track of its location; however, for convenience, it is useful to denote the elevation of the free surface by $\zeta(a, b, t)$, i.e.

$$
\zeta(a, b, t) \equiv z(a, b, 0, t) \text {. }
$$

To obtain non-dimensional variables which are of the order of unity, primed variables are introduced to denote the deviation of each dependent variable from its reference value: $\left(x^{\prime}, y^{\prime}, z^{\prime}\right) \equiv(x, y, z)-(a, b, c)$ and $p^{\prime} \equiv p+\rho g c$. The following nondimensional variables are obtained, based on linear non-dispersive wave theory:

$$
\begin{aligned}
(x, y) & =\left(x^{*}, y^{*}\right) / l, & z & =z^{*} / h_{0}, \quad p=p^{*} / \rho g h_{0} \\
(a, b) & =\left(a^{*}, b^{*}\right) / l, & c & =c^{*} / h_{0}, \\
\left(x^{\prime}, y^{\prime}\right) & =\left(x^{*}, y^{*}\right) h_{0} / l H, & z^{\prime} & =z^{*} / H, \quad p^{\prime}=p^{*} / \rho g H, \\
t & =t^{*}\left(g h_{0}\right)^{\frac{1}{2}} / l, & h & =h^{*} / h_{0},
\end{aligned}
$$

where the superscript $*$ refers to the original dimensional quantities. Here $H$ is a characteristic wave height, $l$ is a characteristic horizontal lengthscale, $h_{0}$ is a characteristic water depth, and $g$ is the acceleration due to gravity. The still water depth is $h$ and $t$ is the time. After substituting these non-dimensional variables into the three-dimensional equations and the boundary conditions the following two dimensionless parameters arise:

$$
\alpha \equiv H / h_{0}, \quad \beta \equiv\left(h_{0} / l\right)^{2} .
$$

The parameter $\alpha$ is a measure of the importance of nonlinear (finite amplitude) effects and $\beta$ is a measure of the importance of frequency dispersion. The analysis here assumes that $\alpha$ and $\beta$ are small, but not infinitesimal, and that $\beta=O(\alpha)$. Following an approach similar to that which was used by Peregrine (1967) to derive Boussinesq type long-wave equations in the Eulerian description, the primed variables are now expressed as power series expansions in the nonlinear parameter $\alpha$ :

$$
x^{\prime}=\sum_{n=0}^{\infty} \alpha^{n} x_{n}^{\prime}, \quad y^{\prime}=\sum_{n=0}^{\infty} \alpha^{n} y_{n}^{\prime}, \quad z^{\prime}=\sum_{n=0}^{\infty} \alpha^{n} z_{n}^{\prime}, \quad p^{\prime}=\sum_{n=0}^{\infty} \alpha^{n} p_{n}^{\prime} .
$$


Under the assumption that $\beta=O(\alpha)$, and that the horizontal components of vorticity are zero, terms of similar order in $\alpha$ are collected after the equations are integrated from $c=-h(a, b)$ to the surface: $c=0$. These irrotationality assumptions are consistent provided the initial and boundary conditions do not introduce vorticity, since it is known that under these conditions the inviscid three-dimensional equations of motion permit only irrotational solutions (Meyer 1971), provided the free surface does not intersect itself. The irrotationality assumptions simplify the analysis by attributing shear production across the depth of the fluid to only nonhydrostatic processes, and in the long-wave limit this shear vanishes; see Zelt (1986) for the details. No assumption is made about the vertical component of vorticity. To simplify the presentation, vector notation will be used, e.g. $\boldsymbol{x}^{\prime}=\left(x^{\prime}, y^{\prime}\right), a=(a, b)$, and $\boldsymbol{\nabla}=(\partial / \partial a, \partial / \partial b)$. The $O\left(\alpha^{0}\right)$ terms yield :

$$
\zeta_{0}=-\nabla \cdot\left(h x_{0}^{\prime}\right), \quad \frac{\partial^{2} x_{0}^{\prime}}{\partial t^{2}}+\nabla \zeta_{0}=0
$$

where $x_{0}^{\prime}$ is independent of $c$. Here $\zeta_{0} \equiv z_{0}^{\prime}(a, 0, t)$. The $O(\alpha)$ terms yield:

$$
\begin{gathered}
\zeta_{1}=-\frac{1}{2} x_{0}^{\prime} \cdot \boldsymbol{H} \cdot x_{0}^{\prime}-\nabla \cdot\left[\left.h x_{1}^{\prime}\right|_{c=0}\right]-\nabla \cdot x_{0}^{\prime}\left[\zeta_{0}+\nabla h \cdot x_{0}^{\prime}\right]-h \frac{\partial\left(x_{0}^{\prime}, y_{0}^{\prime}\right)}{\partial(a, b)} \\
-\frac{\beta}{\alpha} \nabla \cdot\left\{\frac{1}{2} h^{2} \nabla\left[\nabla \cdot\left(h x_{0}^{\prime}\right)\right]-\frac{1}{2} h^{3} \nabla\left(\nabla \cdot x_{0}^{\prime}\right)\right\} \\
\left.\frac{\partial^{2} x_{1}^{\prime}}{\partial t^{2}}\right|_{c=0}+\nabla \zeta_{1}-\left(\nabla \cdot x_{0}^{\prime}\right) \nabla \zeta_{0}+\nabla \zeta_{0} \cdot D_{0}^{\prime}=0
\end{gathered}
$$

Here $\boldsymbol{D}^{\prime}$ and $\boldsymbol{H}$ can be represented in this coordinate system as

$$
\boldsymbol{D}^{\prime} \equiv\left(\begin{array}{cc}
\partial y^{\prime} / \partial b & -\partial x^{\prime} / \partial b \\
-\partial y^{\prime} / \partial a & \partial x^{\prime} / \partial a
\end{array}\right), \quad \boldsymbol{H} \equiv\left(\begin{array}{cc}
\partial^{2} h / \partial a^{2} & \partial^{2} h / \partial a \partial b \\
\partial^{2} h / \partial b \partial a & \partial^{2} h / \partial b^{2}
\end{array}\right)
$$

so that, e.g.

$$
\boldsymbol{\nabla} \zeta_{0} \cdot \boldsymbol{D}_{0}^{\prime} \equiv\left(\frac{\partial\left(\zeta_{0}, y_{0}^{\prime}\right)}{\partial(a, b)}, \frac{\partial\left(x_{0}^{\prime}, \zeta_{0}\right)}{\partial(a, b)}\right)
$$

One way to proceed is to solve the zero-order equations, substitute these solutions into the first-order equations, and then solve these. As discussed by Peregrine (1967), such a procedure will yield a solution valid only for small time. A better way to proceed is to combine the zero-order equations with the first-order equations to obtain a single set of equations for variables that includes both the zero-order and the first-order effects. These variables are chosen to be

$$
\eta(a, t) \equiv \zeta_{0}(a, t)+\alpha \zeta_{1}(a, t), \quad \bar{x}(a, t) \equiv \frac{1}{h(a)} \int_{-h(a)}^{0}\left\{x_{0}^{\prime}(a, c, t)+\alpha x_{1}^{\prime}(a, c, t)\right\} \mathrm{d} c .
$$

Clearly $\eta=\zeta+O\left(\alpha^{2}\right)$. The vector $\bar{x}$ corresponds to 'depth averaged' fluid displacements, although the integration is over the Lagrangian coordinate $c$, not $z$. With this choice of variables, the zero and first-order terms in (2), (3), and (4), can be combined to yield equations for $\eta$ and $\bar{x}$ on which a finite element model can be based:

$$
\eta=-\nabla h \cdot \overline{\boldsymbol{x}}-\frac{1}{2} \alpha \overline{\boldsymbol{x}} \cdot \boldsymbol{H} \cdot \overline{\boldsymbol{x}}-h \frac{\left[\boldsymbol{\nabla} \cdot \overline{\boldsymbol{x}}+\alpha \frac{\partial(\bar{x}, \bar{y})}{\partial(a, b)}\right]}{1+\alpha \boldsymbol{\nabla} \cdot \overline{\boldsymbol{x}}},
$$




$$
(1+\alpha \boldsymbol{\nabla} \cdot \overline{\boldsymbol{x}}) \frac{\partial^{2} \bar{x}}{\partial t^{2}}+\alpha \nabla \eta \cdot \overline{\boldsymbol{D}}+\boldsymbol{\nabla} \eta=\beta\left\{\frac{1}{2} h \boldsymbol{\nabla}\left[\boldsymbol{\nabla} \cdot\left(h \frac{\partial^{2} \bar{x}}{\partial t^{2}}\right)\right]-\frac{1}{6} h^{2} \nabla\left(\boldsymbol{\nabla} \cdot \frac{\partial^{2} \bar{x}}{\partial t^{2}}\right)\right\}
$$

These equations are equivalent to the long-wave equations in the Eulerian description derived by Peregrine (1967). They are accurate to $O(\alpha, \beta)$; however, the nonlinear and dispersive terms can be altered using the zero-order relations of (2). Notice that to the same order of approximation, (5) also can be written as:

$$
\alpha \eta=\frac{h(a)}{1+\alpha \boldsymbol{\nabla} \cdot \bar{x}+\alpha^{2} \frac{\partial(\bar{x}, \bar{y})}{\partial(a, b)}}-h(a+\alpha x)
$$

which can be obtained easily using a simple control volume argument.

\subsection{Finite element formulation}

The finite element model is based on the Galerkin weighted residual method (Connor $\&$ Brebbia 1976). Equation (6) is multiplied by weighting functions $\hat{x}$ and $\hat{y}$ and then integrated over the domain of the problem, $\Omega$ :

$$
\begin{aligned}
\iint_{\Omega}\{(1+\alpha \boldsymbol{\nabla} \cdot \boldsymbol{x}) & \left.\frac{\partial^{2} \boldsymbol{x}}{\partial t^{2}} \cdot \hat{\boldsymbol{x}}+\alpha \boldsymbol{\nabla} \eta \cdot \boldsymbol{D} \cdot \hat{\boldsymbol{x}}+\boldsymbol{\nabla} \eta \cdot \hat{\boldsymbol{x}}\right\} \mathrm{d} a \mathrm{~d} b \\
& -\beta \iint_{\Omega}\left\{\frac{1}{2} h \boldsymbol{\nabla}\left[\boldsymbol{\nabla} \cdot\left(h \frac{\partial^{2} x}{\partial t^{2}}\right)\right]-\frac{1}{6} h^{2} \nabla\left(\nabla \cdot \frac{\partial^{2} x}{\partial t^{2}}\right)\right\} \cdot \hat{\boldsymbol{x}} \mathrm{d} a \mathrm{~d} b=0,
\end{aligned}
$$

where $\hat{\boldsymbol{x}}=(\hat{x}, \hat{y})$ and the bars have been dropped from the particle displacements to simplify the notation. Green's first identity is applied to this integral to remove all second-order spatial derivatives. This generates integrals along the boundary of the domain, $\partial \Omega$, which can be used to impose boundary conditions for the problem. All spatial derivatives of $\eta$ also can be eliminated using Green's first identity, although this procedure generates a term containing $\boldsymbol{\nabla} \cdot \boldsymbol{D}$, which involves second-order spatial derivatives of $\boldsymbol{x}$. However, from the definition of $\boldsymbol{D}, \boldsymbol{\nabla} \cdot \boldsymbol{D} \equiv \mathbf{0}$, so (5) can be substituted into the weighted residual integral to eliminate $\eta$ without generating second-order spatial derivatives, resulting in the following:

$$
\begin{aligned}
0= & \iint_{\Omega}(1+\alpha \nabla \cdot \boldsymbol{x}) \frac{\partial^{2} \boldsymbol{x}}{\partial t^{2}} \cdot \hat{\boldsymbol{x}} \mathrm{d} a \mathrm{~d} b \\
& +\iint_{\Omega} \boldsymbol{\nabla} h \cdot \boldsymbol{x}+\frac{1}{2} \alpha \boldsymbol{x} \cdot \boldsymbol{H} \cdot \boldsymbol{x}+h \frac{\left[\boldsymbol{\nabla} \cdot \boldsymbol{x}+\alpha \frac{\partial(x, y)}{\partial(a, b)}\right]}{1+\alpha \boldsymbol{\nabla} \cdot \boldsymbol{x}}(\boldsymbol{\nabla} \cdot \hat{\boldsymbol{x}}+\alpha \boldsymbol{D}: \boldsymbol{\nabla} \hat{\boldsymbol{x}}) \mathrm{d} a \mathrm{~d} b \\
& +\beta \iint_{\Omega}\left\{\frac{1}{3} h^{2} \nabla \cdot \frac{\partial^{2} \boldsymbol{x}}{\partial t^{2}} \boldsymbol{\nabla} \cdot \hat{\boldsymbol{x}}+\frac{1}{6} h \boldsymbol{\nabla} \cdot \frac{\partial^{2} \boldsymbol{x}}{\partial t^{2}} \boldsymbol{\nabla} \cdot \hat{\boldsymbol{x}}+\frac{1}{2} h \boldsymbol{\nabla} h \cdot \frac{\partial^{2} \boldsymbol{x}}{\partial t^{2}} \boldsymbol{\nabla} \cdot \hat{\boldsymbol{x}}+\frac{1}{2} \nabla h \cdot \frac{\partial^{2} \boldsymbol{x}}{\partial t^{2}} \boldsymbol{\nabla} h \cdot \hat{\boldsymbol{x}}\right\} \mathrm{d} a \mathrm{~d} b \\
& -\beta \int_{\partial \Omega}\left\{\frac{1}{3} h^{2} \nabla \cdot \frac{\partial^{2} \boldsymbol{x}}{\partial t^{2}}+\frac{1}{2} h \boldsymbol{\nabla} h \cdot \frac{\partial^{2} \boldsymbol{x}}{\partial t^{2}}\right\} \hat{\boldsymbol{x}} \cdot \boldsymbol{n} \mathrm{d} s+\int_{\partial \Omega} \eta[\alpha \boldsymbol{n} \cdot \boldsymbol{D} \cdot \hat{\boldsymbol{x}}+\hat{\boldsymbol{x}} \cdot \boldsymbol{n}] \mathrm{d} s,
\end{aligned}
$$

where $\boldsymbol{n}$ is the outward unit normal vector to the domain $\Omega$ and where the two vertical dots refer to the Gibbs' double-dot product:

$$
\boldsymbol{D}: \boldsymbol{\nabla} \hat{\boldsymbol{x}}=D_{i j} \frac{\partial \hat{x}_{j}}{\partial \boldsymbol{a}_{i}}
$$

with summation over all free indices. 


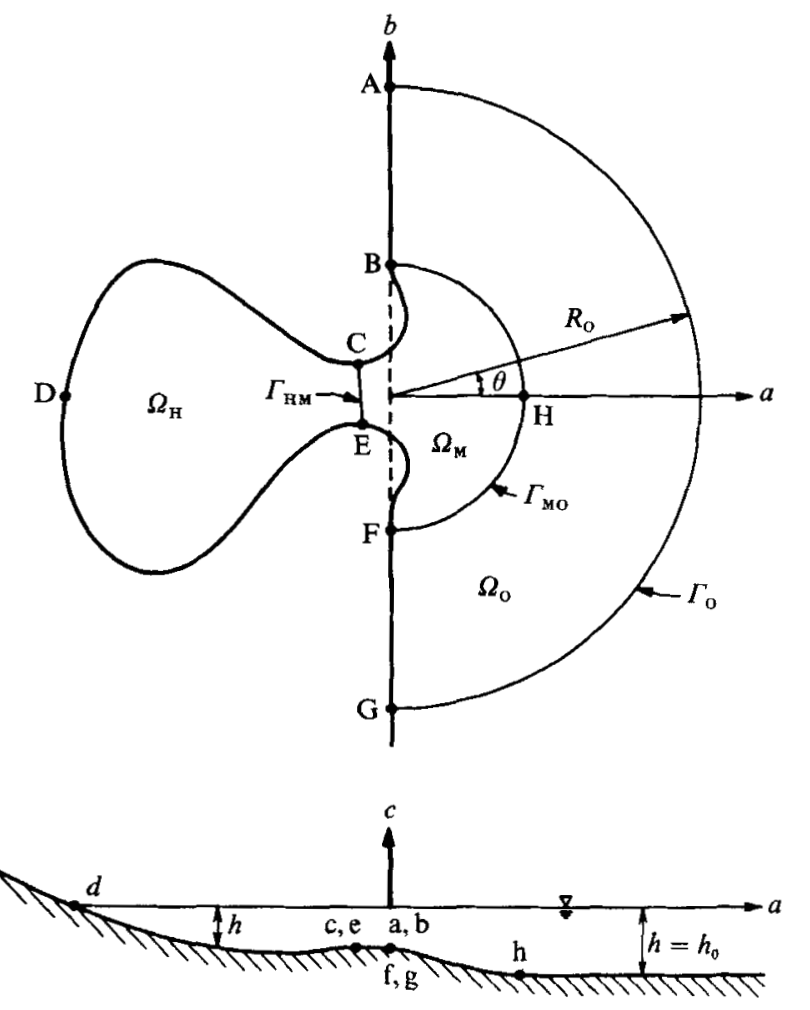

Figure 1. Definition sketch of the harbour region $\Omega_{\mathrm{H}}$, the harbour mouth region $\Omega_{\mathrm{M}}$, and the outer region $\Omega_{\mathrm{o}}$.

The final procedure is to discretize the weighted residual formulation to transform it into a matrix problem with a finite number of unknowns. Isoparametric elements were chosen to discretize arbitrary two-dimensional regions, which in the case of (7) leads to a system of algebraic equations that can be written in matrix form as: $\boldsymbol{M} \ddot{d}=f$, where the dots represent time derivatives of the components of $d$. The vector $\boldsymbol{d}$ contains the particle displacement nodal unknowns :

$$
d(t)=\left(x_{1} y_{1} x_{2} y_{2} \ldots x_{N} y_{N}\right)^{\mathrm{T}} \quad(N=\text { number of nodes }),
$$

where the $\mathrm{T}$ denotes matrix transpose. To simplify the presentation, dissipation has been neglected here; it is introduced in $\$ 2.3$ where harbour entrance losses and a radiation boundary condition are discussed. With dissipation included, (7) generalizes to: $\boldsymbol{M} \ddot{d}+\boldsymbol{C} \dot{d}=f$. Details of the discretization, including dissipation are given in the Appendix.

\subsection{Harbour response formulation}

Figure 1 illustrates schematically a general formulation applicable to harbour resonance studies. Henceforth, all variables will be dimensional. The domain $\Omega$, representing the locations of the fluid particles at time $t=0$, is comprised of the union of three non-overlapping regions $\Omega_{\mathrm{H}}, \Omega_{\mathrm{M}}$, and $\Omega_{\mathrm{O}}$, i.e. $\Omega=\Omega_{\mathrm{H}} \cup \Omega_{\mathrm{M}} \cup \Omega_{\mathrm{O}}$. The region $\Omega_{\mathrm{H}}$ is the inner harbour region bounded by the harbour entrance $\Gamma_{\mathrm{HM}}$ and the curve $\mathrm{CDE}$. In the definition sketch, the water depth is zero at the shore line, but 
the harbour also may be bounded by vertical walls or by a combination of vertical walls and sloping boundaries. It is assumed that all energy is reflected by the boundary CDE, whether or not the walls are vertical. The region $\Omega_{\mathrm{M}}$ is the area comprising the harbour mouth out to the boundary $\Gamma_{\text {MO }}$. The outermost region $\Omega_{\mathrm{O}}$ is bounded by $\Gamma_{\text {MOO }}, \Gamma_{\mathrm{O}}$, and the physical boundaries $A B$ and $F G$. This region models the open ocean. The boundaries $\mathrm{AB}$ and FG are assumed to be vertical and perfectly reflecting, and the water depth is constant and equal to $h_{0}$.

\subsubsection{Boundary conditions}

Along open boundaries with a non-zero water depth the wave height can be specified as a function of time, i.e.

$$
\eta=\eta_{\mathrm{s}}(\boldsymbol{a}, t) .
$$

The function $\eta_{\mathrm{s}}$ is in the Lagrangian description; therefore, if the wave height is known from Eulerian wave gauge recordings, it must be transformed to the Lagrangian frame of reference before this boundary condition can be applied. However, if the wave height is small along the boundary, then nonlinear effects can be neglected and the Eulerian record can be substituted for the Lagrangian record with negligible error. This boundary condition is imposed by substituting (8) for $\eta$ into the last boundary integral of (7) along the portion of the boundary where it applies.

The boundaries, $\Gamma_{\mathrm{MO}}$ and $\Gamma_{\mathrm{HM}}$ are special internal boundaries. An incident wave of the form: $\eta=\eta_{\text {inc }}\left(t+a\left(g h_{0}\right)^{\frac{1}{2}}\right)$ is introduced along the internal boundary $\Gamma_{\text {MO }}$ propagating toward the harbour entrance. In the absence of the harbour (dashed line in figure 1) the wave reflected from the coast is approximately $\eta=\eta_{\text {inc }}\left(t-a\left(g h_{0}\right)^{\frac{1}{2}}\right)$, although nonlinear and dispersive effects will alter this somewhat. This estimate of the reflected wave is subtracted from waves crossing $\Gamma_{\text {Mo }}$ into the outer region, leaving only waves generated by the presence of the harbour itself. The radiation boundary condition studied by Mungall \& Reid (1978), here expressed in the Lagrangian description, is used to absorb these radiated waves along $\Gamma_{\mathrm{o}}$ :

$$
\frac{\eta}{h_{0}}=\frac{1}{\left(g h_{0}\right)^{\frac{1}{2}}} \frac{\partial r}{\partial t}-\frac{r}{2 R_{\mathrm{O}}},
$$

where $r=x \cos \theta+y \sin \theta$ is the radial component of displacement of a fluid particle, and $R_{\mathrm{O}}$ is the distance from the harbour entrance to the boundary (see figure 1 ). The decomposition of the solution into a radiated wave and an incident-reflected wave system ignores nonlinear effects. Therefore, the boundary $\Gamma_{\text {MO }}$ must be chosen sufficiently far from the entrance so that nonlinear effects are small and linear superposition is valid.

Boundary $\Gamma_{\mathrm{HM}}$ is located in the entrance region where significant dissipation can occur, especially near resonance when the horizontal velocity is large. A head loss quadratic in the velocity is employed, i.e. $\Delta \eta=(f / 2 g) U \mid U$, where $\Delta \eta$ is the change in the surface elevation across the constriction, $f$ is an entrance loss coefficient $(\approx 1)$, and $U$ is the signed horizontal velocity normal to $\Gamma_{\mathrm{HM}}$. This parametrization has been used successfully by Mei, Liu \& Ippen (1974), Ünlüata \& Mei (1975), Rogers \& Mei (1978), Lepelletier (1980) and others.

Along an impermeable vertical wall, the motion of a fluid particle must be perpendicular to the outward wall normal, $n$, at the present position of the particle:

$$
\frac{\partial \boldsymbol{x}}{\partial t} \cdot \boldsymbol{n}(\boldsymbol{a}+\boldsymbol{x})=0 .
$$




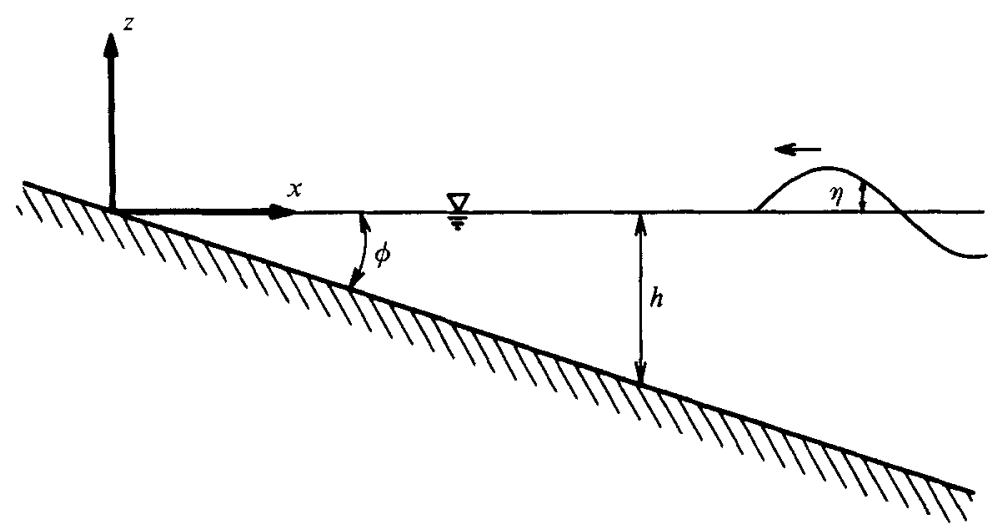

Figure 2. Definition sketch for the run-up of waves on a sloping boundary.

Note that as the particle moves, the outward normal will in general change direction if the wall is curved. Therefore, this expression cannot be integrated to yield $\boldsymbol{x} \cdot \boldsymbol{n}=\boldsymbol{0}$ (the details of the treatment of this boundary condition are discussed in the Appendix).

Along a sloping boundary where the still water depth decreases continuously to zero, the water surface elevation is related to the still water depth as:

$$
\eta=-h(a+x)
$$

Like (8), this boundary condition is imposed by substituting (11) for $\eta$ into the last boundary integral of (7) along the boundary where it applies. Since the treatment of sloping boundaries by the finite element model is an important aspect of this work, it is discussed here in some detail and the results of the model are compared to a theoretical solution for this process. Exact solutions to nonlinear wave theories are rare, especially if the fluid boundaries are free to deform with the run-up of waves on a beach. For that reason, the solution obtained by Carrier \& Greenspan (1958) is remarkable. It is an exact solution of the inviscid nonlinear shallow-water equations (neglecting frequency dispersion) for normal incidence of the run-up of non-breaking periodic waves on a plane beach. It is the ideal solution to test the ability of this finite element model to handle the nonlinear aspects of the interaction of long waves with a sloping boundary.

A definition sketch for the run-up of waves on a beach with beach angle $\phi$ is presented in figure 2. This is a special case of figure 1 where the harbour and mouth regions, $\Omega_{\mathrm{H}}$ and $\Omega_{\mathrm{M}}$, form a channel with straight parallel sides and a linearly varying depth; the outer region, $\Omega_{\mathrm{O}}$, with its radiation boundary, has been neglected. Entrance dissipation is also neglected since the channel width is constant. Since shoaling decreases the local wavelength scale as a wave approaches the shore, the node spacing of the finite element grid was decreased in this direction accordingly. The grid consisted of 57 parabolic quadrilateral elements, where the node spacing increased by a factor of seven from the shore to the offshore boundary to provide high resolution at the shore without incurring a timestep penalty if the same resolution were used in the deeper water. The shore was located at $a=0$ and the offshore boundary at $a=a_{\max }$. The Carrier-Greenspan solution is a steady-state solution, and to compare it with the results of the finite element model, the fluid was assumed to 
be quiescent for $t<0$; for $t>0$ the surface elevation was prescribed at the offshore boundary as:

$$
\eta=\operatorname{Re}\left\{\frac{1}{2} A_{0} H_{0}^{(1)}\left(2 \omega\left(\frac{a_{\max }}{g \phi}\right)^{\frac{1}{2}}\right) \mathrm{e}^{\mathrm{i} \omega(t+\delta)}\right\} F(t),
$$

where $\omega$ is the temporal frequency, $A_{0}$ is an amplitude parameter discussed below, $H_{0}^{(1)}$ is the Hankel function of the first kind of order zero, and Re refers to the real part of a complex quantity. The phase, $\delta$, was chosen so that $\eta=0$ at $t=0$. The function $F(t)$, equal to $1-\mathrm{e}^{-0.2(\omega t)^{2}}$, ensures the particle acceleration is zero at $t=0$. The wave specified by (12) reflects from the shore at $a=0$ and propagates back toward the offshore boundary at $a=a_{\max }$, setting up the standing wave solution obtained by Carrier \& Greenspan (1958). The vertical excursion of the shore line is $\pm A_{0}$ according to both linear theory and the nonlinear Carrier-Greenspan solution. Since a radiation boundary was not used, the wave which propagates toward the offshore boundary reflects back into the computational domain, changing the standing wave system, so the simulation must stop before this wave contaminates the region of interest.

The appropriate horizontal lengthscale near the shore is $l=g \phi / \omega^{2}$, the water depth scale is $h_{0}=\phi l$, and the wave elevation scale is $H=A_{0}$. Hence, the parameter which characterizes the nonlinearity of the solution is $\alpha \equiv H / h_{0}=A_{0} \omega^{2} / g \phi^{2}$. An important result obtained from the Carrier-Greenspan solution is that a physically reasonable steady-state solution of the inviscid shallow water equations exists only for : $\alpha \leqslant 1$. Nonlinear effects cause the wavefront to steepen at the shore line as the amplitude of the incident wave increases. For $\alpha=1$ the tangent to the water surface becomes vertical at the run-down point. For $\alpha>1$ the solution becomes multivalued and cannot be computed with the finite-element model since the Jacobian of the transformation between the $(a, b)$ and the $(x, y)$ coordinates must remain positive; this regime is discussed below.

Figure $3(a)$ shows the elevation of the shore line as a function of time as computed by the finite-element model for six different values of the nonlinear parameter $\alpha$. The offshore boundary was set at $a_{\max }=95 l$ and a time step $\Delta t=0.05 / \omega$ was chosen. The lowest amplitude run-up record corresponds to $\alpha=0.2$ and in order of increasing amplitude the other records shown by solid curves correspond to $\alpha=0.5,0.8,0.9$ and 1.0 ; the dashed curve corresponds to $\alpha=1.15$. The shore line is quiescent until the wave arrives at about $\omega t=19$. The wave reflected from the outer computational boundary returns at about three times this value, near $t=57$; thus, the abscissa must be limited to about $\omega t<54$.

The numerical solution for the case $\alpha=1.15$ (dashed curve) could only be computed until approximately $t=23.5$, at which point the wave had reached the point of maximum run-down and the Jacobian of the transformation between the Lagrangian and the Eulerian frames at the shore line dropped below a small tolerance parameter (which had been arbitrarily set to 0.01 in the numerical model), signifying that the water surface was almost vertical and about to become multivalued. Theoretically, a vertical tangent should have occurred for $\alpha=1$, but since the finite element grid has a finite resolution near the shore, it is difficult to compute the large gradient accurately. Two periods of the motion in figure $3(a)$, from about $\omega t=39$ to $\omega t=52$, are compared to the theory of Carrier \& Greenspan (1958) in figure $3(b)$. The agreement is surprisingly good, even for the case $\alpha=1$, and it is difficult to differentiate between the theory and the results of the finite element model. 

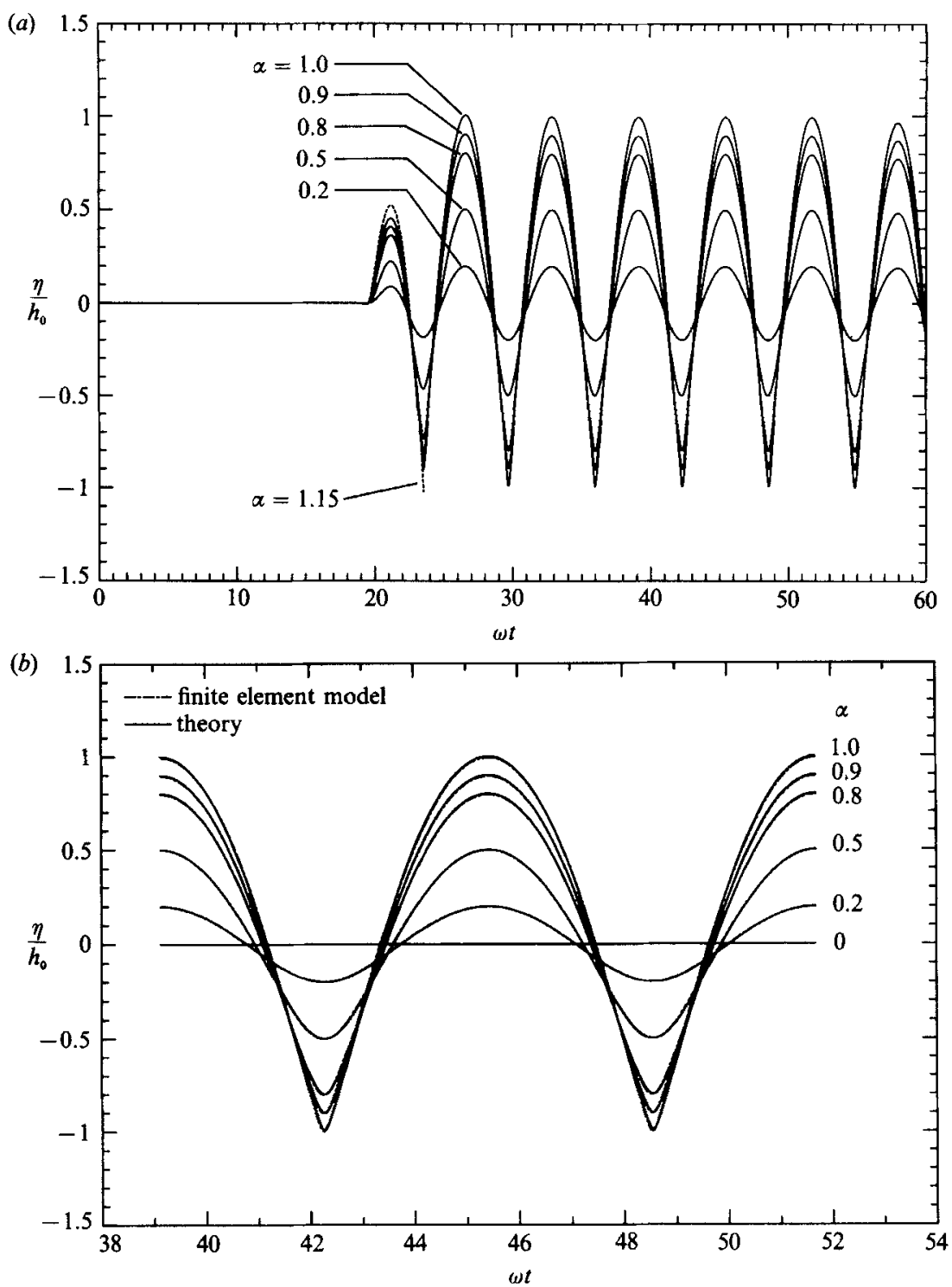

FraURe 3. (a) Shore line elevation computed by the finite element model for: $\alpha=0.2,0.5,0.8,0.9$, 1.0, 1.15. (b) Two periods compared with steady-state theory of Carrier \& Greenspan $(1958) ;-.-$, finite element model; - $\longrightarrow$, theory.

Figure 4 contrasts the wave profiles predicted by linear theory $(\alpha=0.01)$ and nonlinear theory $(\alpha=1)$ as computed by the finite element model and the exact solution of Carrier \& Greenspan (1958). The Carrier-Greenspan solution is plotted with solid lines for the times $\omega t=\frac{1}{4} \mathrm{i} \pi, i=0,1,2,3,4$, and the numerical results are plotted with open circle symbols for the times: $\omega t=39.1,39.9,40.7,41.5$ and 42.25 (temporal interpolation was not performed). By following the symbols plotted for the finite element solution, one can follow the trajectories of the particles through the fluid. Near $x / l=1.4$, where a node exists in the linear theory, the nonlinear theory actually exhibits two maxima and minima per wave period; the maxima occur at the 

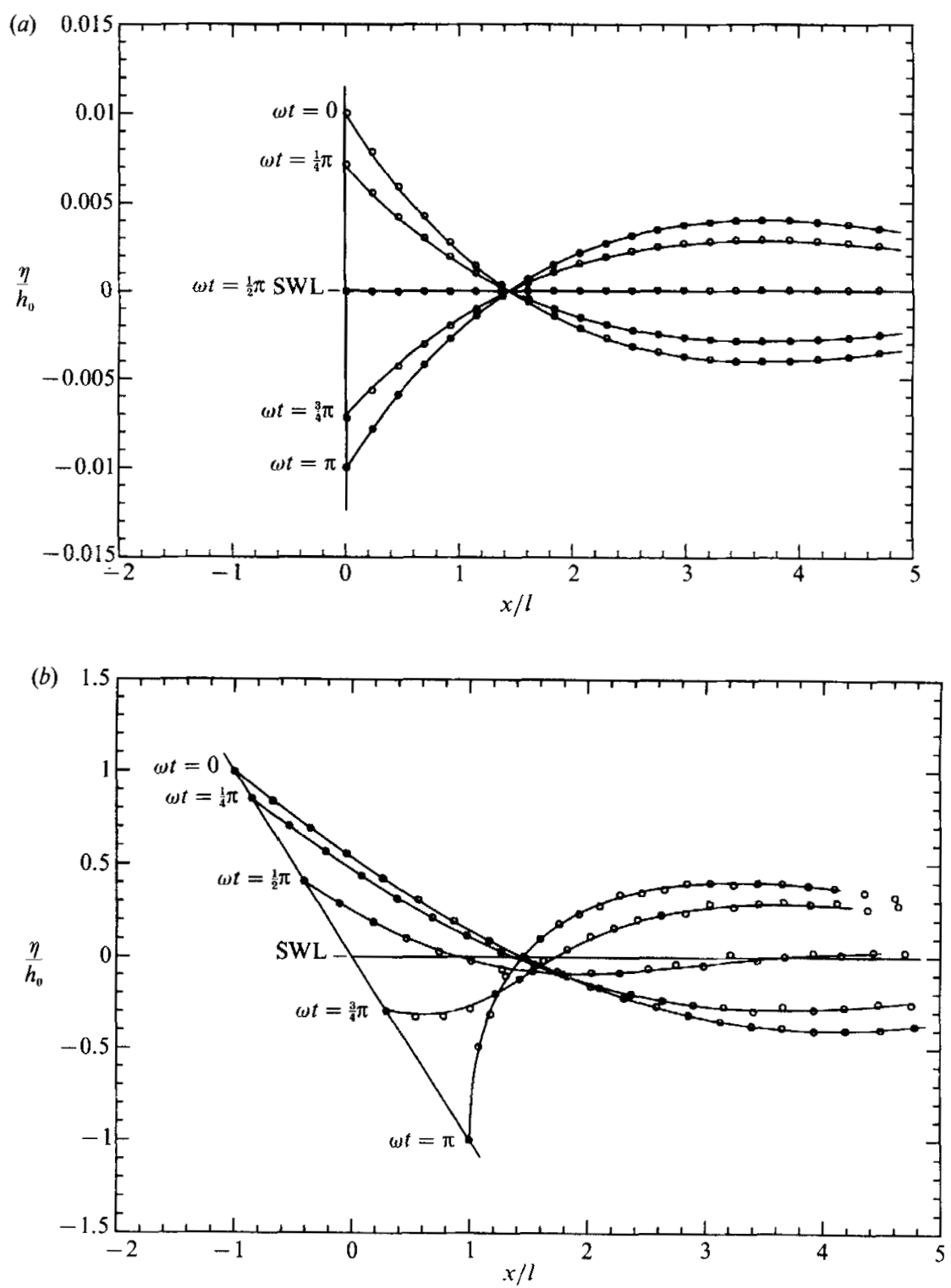

Figure 4. Comparison between the wave profiles as predicted by $O$, the finite element model, and - the theory of Carrier \& Greenspan $(1958) ;(a) \alpha=0.01 ;(b) \alpha=1.0$.

times of maximum run-up and maximum run-down. Agreement between the numerical model and the Carrier-Greenspan solution is very good for $\alpha=1.0$, except for slight oscillations in the surface profile with a wavelength roughly equal to twice the grid spacing. Up to $\alpha=0.9$ the numerical results were smooth; however, for $\alpha>0.9$ these oscillations appeared at the time of the first run-down trough shown in figure $3(a)$, at about $\omega t=23.5$, the time of maximum acceleration at the shore line. However, these high-frequency oscillations did not grow noticeably over the duration of the simulation, and probably could be reduced by using a more stable time integration scheme. 
According to the Carrier-Greenspan solution, at the point of maximum run-down the horizontal fluid acceleration is (Zelt 1986):

$$
\frac{\mathrm{d} u}{\mathrm{~d} t}=-\frac{\alpha}{1-\alpha}
$$

Thus, as $\alpha \rightarrow 1$ it is not surprising that the numerical model encounters a problem. Since the horizontal acceleration grows without bound, then so also must the vertical acceleration. However, this contradicts the hydrostatic assumption upon which the Carrier-Greenspan solution is based, and indicates that non-hydrostatic pressure effects should be considered in the theory. On the other hand, the inclusion of nonhydrostatic effects is not sufficient to prevent wave breaking and the associated numerical problem of encountering a zero Jacobian, as discussed above. Some authors (Tuck \& Hwang 1972; Synolakis 1987) have discussed the desirability of advancing the solution through time while the Jacobian is negative, because the solution so obtained when the Jacobian returns positive may be useful for studying breaking and near-breaking waves. Such a procedure is possible under a suitable change of coordinates, but not with the Lagrangian finite element model described herein. It is possible to artificially place a lower bound on the value of the Jacobian used in the numerical calculation, but the implications of doing this are not clear. A better way to proceed might be to introduce artificial dissipation in regions of high surface slope (small Jacobian) to simulate the energy loss during breaking and to retain a positive Jacobian.

\subsubsection{Frequency dispersion}

In order to investigate the ability of the finite element model to compute nonhydrostatic pressure effects not treated by traditional long-wave models, numerical experiments were conducted where waves of a single harmonic frequency were generated in a long channel of constant depth $h$ and straight parallel sides. Nonlinear effects were neglected to isolate the effects of frequency dispersion from those due to amplitude dispersion, and the phase speed was determined numerically for different frequencies. The dispersion relation for the weakly dispersive theory presented in $\S 2.1$, on which the finite element model is based, can be obtained by substituting a wave of the form $\eta \sim \mathrm{e}^{\mathrm{i}(k a-\omega t)}$ into (5) and (6), neglecting nonlinear terms. The result is

$$
\omega_{\mathrm{wd}}^{2}=\frac{g h k^{2}}{1+\frac{1}{3}(k h)^{2}} .
$$

where the subscript wd refers to the weakly dispersive wave theory of $\$ 2.1$. The corresponding phase speed, $c_{\mathrm{wd}} \equiv \omega_{\mathrm{wd}} / k$, can then be compared to expressions for $c_{\mathrm{fd}}$ and $c_{\text {nd }}$, the phase speeds for fully dispersive linear theory, and non-dispersive linear theory, respectively:

$$
\begin{aligned}
c_{\mathrm{fd}} & =(g h)^{\frac{1}{2}}(\tanh k h / k h)^{\frac{1}{2}}, \\
c_{\mathrm{wd}} & =(g h)^{\frac{1}{2}}\left[1+\frac{1}{3}(k h)^{2}\right]^{-\frac{1}{2}}, \\
c_{\mathrm{nd}} & =(g h)^{\frac{1}{2}} .
\end{aligned}
$$

The results of the finite element model are compared to these three theories in figure 5. As expected, the finite element model results agree very well with the weakly dispersive theory from which the model was developed. Also plotted in figure 5 is the classical long-wave limit. This is not a precisely defined quantity, but it is generally agreed to correspond to approximately $h / \lambda \approx 0.05$ (Eagleson $\&$ Dean 1966), where 


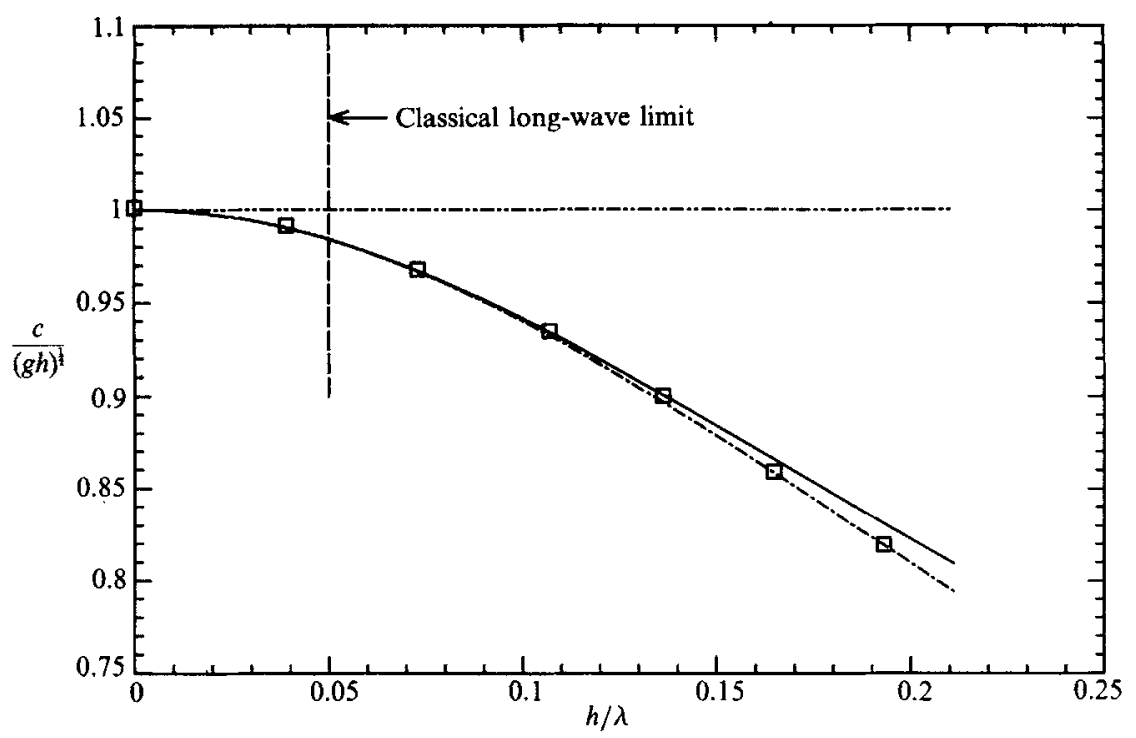

Figure 5. Wave celerity computed with the finite element model compared to three linear wave theories (see text for details). $\square$, finite element method;,$- c_{\mathrm{fd}}-$ full dispersive theory; - . - . $c_{\mathrm{wd}}-$ weakly dispersive long-wave theory $;-\cdots, c_{\mathrm{nd}}$-non-dispersive long-wave theory.

$\lambda=2 \pi / k$. Beyond this value, the error between non-dispersive long-wave theory (the horizontal dashed line) and fully dispersive theory (solid line) grows unacceptable for some applications. However, from this figure it can be seen that the weakly dispersive theory (and the finite element model) is more accurate and can be applied to much larger values of $h / \lambda$; the error in the wave celerity for $h / \lambda=0.2$ with the weakly dispersive theory and the finite element model is roughly the same as that for $h / \lambda=0.05$ with the non-dispersive theory, representing an increase of a factor of four in the range of wavenumbers for accurately computing the phase speed.

\section{Harbour response experiments}

To compare the results of the finite element model with laboratory experiments, the harbour region $\Omega_{\mathrm{H}}$ in figure 1 was specialized to a long rectangular basin where the depth decreased linearly from the entrance to the shore line. A sketch of this case and a grid used by the finite element model is shown in figure 6. The laboratory experiments were conducted in a wave basin $4.73 \mathrm{~m}$ wide and $9.6 \mathrm{~m}$ long. The still water depth was $h_{0}=7 \mathrm{~cm}$ in the constant-depth outer region representing the open sea, where a train of weakly nonlinear cnoidal waves was produced with a microprocessor-controlled servo-hydraulic wave generator using the nonlinear wave generation scheme developed by Goring (1978). The wave period was $T=2 \mathrm{~s}$, and based on cnoidal wave theory the approximate wavelength was $\lambda=1.64 \mathrm{~m}\left(\lambda / h_{0}=\right.$ 23.4), close to the linear long-wave result computed from $\lambda=T\left(g h_{0}\right)^{\frac{1}{2}}$. The fundamental harmonic lies in the classic long-wave regime, but the higher harmonics do not. The wave generator was located approximately $6.9 \mathrm{~m}$ from the harbour entrance. Waves that propagated away from the harbour eventually reflected from the wave generator and returned to the harbour; therefore, for these experiments it was possible to expose the harbour to only eight periods $(2 \times 6.9 \mathrm{~m} \div 1.64 \mathrm{~m} \approx 8)$ of 


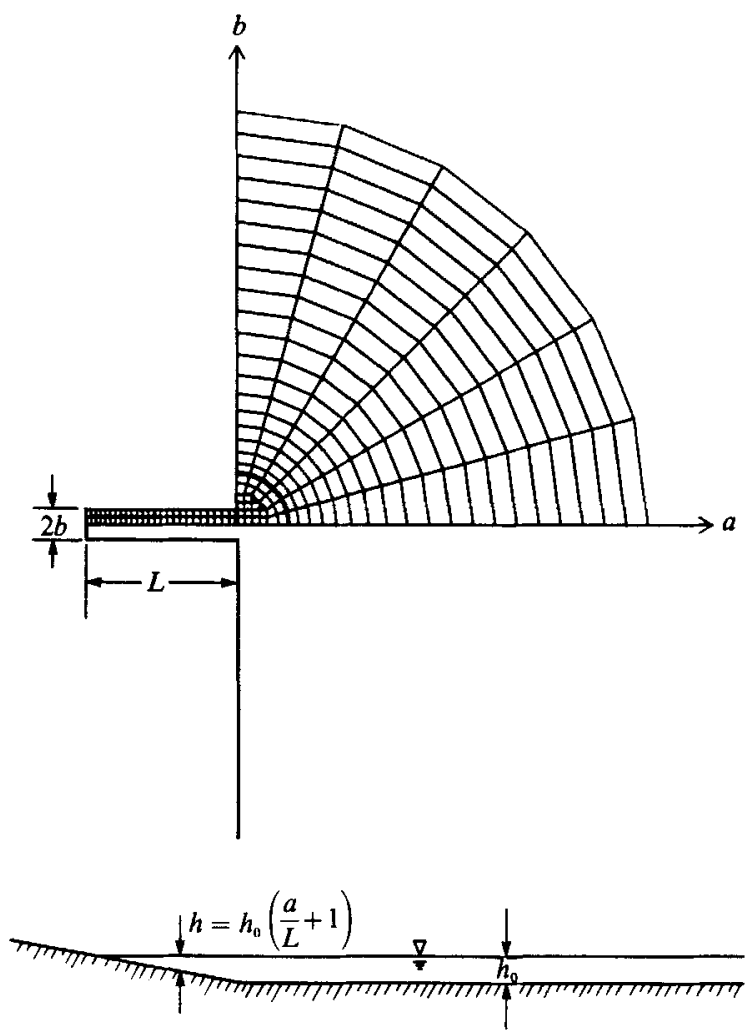

Figure 6. Harbour geometry for laboratory experiments and finite element grid for a rectangular harbour with water depth decreasing linearly to zero from the entrance to the shore line.

the incident wave. The short generation time prevented longitudinal tank modes from developing; transverse paddle modes were not observed.

An important parameter which characterizes the response of a harbour to incident waves is the ratio of a characteristic horizontal length of the harbour to the wavelength of the incident wave. For a rectangular harbour, this can be written as $k L=2 \pi L / \lambda$ where $L$ is the harbour length, and $\lambda$ is the wavelength. For shallow water waves a similar parameter may be written instead in terms of the wave frequency: $\omega L /\left(g h_{0}\right)^{\frac{1}{2}}$. Owing to weakly nonlinear and dispersive effects, $k L$ and $\omega L /\left(g h_{0}\right)^{\frac{1}{2}}$ are not equivalent. For these experiments the parameter $\omega L /\left(g h_{0}\right)^{\frac{1}{2}}$ was chosen as the harbour response parameter because the wave frequency, $\omega$, was a specified parameter, whereas the wavelength, $\lambda$, had to be computed theoretically or measured experimentally. Twenty-one separate experiments were performed with different values of $\omega L /\left(g h_{0}\right)^{\frac{1}{2}}$ varying from 0 to 2.08 , fully encompassing the first resonant mode. For each experiment, the surface displacement was measured as a function of time on the centreline of the harbour midway between the entrance and the quiescent shore line. Resistance wave gauges with an accuracy of $\pm 0.01 \mathrm{~cm}$ were used. (Measurements of the elevation of the shore line as a function of time were not made because of the difficulties in the experimental techniques at the scale of these measurements.) The steady-state peak-to-peak wave amplitude at the harbour entrance with the entrance closed $\left(\omega L /\left(g h_{0}\right)^{\frac{1}{2}}=0\right)$ was $2 H=0.20 \mathrm{~cm}$. The same incident wave was used for each of the experiments so that the spectral energy 
distribution in the incident wave was fixed and the characteristic timescale $T$ was also fixed. The response of the harbour was obtained by changing the length of the harbour and incrementally changing its width to maintain a width to length ratio approximately equal to 0.2 . Thus, the harbour response parameter $\omega L /\left(g h_{0}\right)^{\frac{1}{2}}$ was varied by changing $L$, not $\omega$.

A grid used by the finite-element model is sketched in figure 6 . The grid spacing decreases near the shore to match the reduction of the local wavelength in shallow water. Since the case chosen is symmetric about the centreline of the harbour, the grid covers only half the domain. As mentioned earlier, the ratio of the width of the harbour to its length is 0.2. The inter-region boundary $\Gamma_{\text {MO }}$, defined in figure 6 , is the circular are which has been darkened for better definition, and boundary $\Gamma_{\mathrm{H}}$ is the darkened vertical line across the entrance to the harbour.

To model the entrance dissipation, a value for the entrance loss coefficient of $f=$ 0.8 was chosen based on the work of Lepelletier (1980) for a fully open rectangular harbour with sharp corners at the entrance. Additional boundary dissipation occurs along the bottom in the constant depth and variable depth regions. Experiments by Van Dorn (1966) indicate that a boundary layer also forms quickly at the free surface, even with an initially clean liquid, due to a rapid accumulation of surfactants. However, boundary-layer dissipation was approximately an order of magnitude smaller than that associated with radiation for the experiments described here, so it did not influence the results significantly. Nevertheless, its contribution was modelled and the interested reader is referred to Zelt (1986) for a discussion of the numerical implementation.

Figure 7 compares six of the water surface time histories obtained experimentally with those computed by the finite element model for different values of $\omega L /\left(g h_{0}\right)^{\frac{1}{2}}$. Overall agreement is very good, and it is difficult to differentiate between the results from the experiments and the finite element model. To compare the results of the numerical model with the laboratory experiments, the results of the model were transformed to the Eulerian description; the wave records in figure 7 are in the Eulerian description. The water surface time history with $\omega L /\left(g h_{0}\right)^{\frac{1}{2}}=0$, i.e. the record obtained with no harbour present, was used to define the incident-reflected wave used for input to the finite element model.

The records for $\omega L /\left(g h_{0}\right)^{\frac{1}{2}}=0.70$ and 0.89 correspond to frequencies which are less than that for the first resonant mode. The finite element model results agree extremely well with the experimental results for these cases, with only a slight discrepancy in the minimum wave extrema for $\omega L /\left(g h_{0}\right)^{\frac{1}{2}}=0.89$. The largest response occurs for $\omega L /\left(g h_{0}\right)^{\frac{1}{2}}=1.01$ which corresponds to the first resonant mode of the harbour. The amplitude of the finite element model results is slightly smaller than that of the experimental results but the agreement is still very good. Notice that the troughs are larger than the peaks; in fact, the mean value of the steady-state portion of the wave record is negative. At the point of measurement, where $h=\frac{1}{2} h_{0}$, the crests and troughs in the experimental wave record are approximately $\eta / h_{0}=0.11$ and $\eta / h_{0}=-0.16$, respectively. That is a nonlinear effect for shoaling waves discussed by Zelt (1986), and can be seen in figure $4(b)$ near $x / l=1.45$. The effect is very noticeable near resonance where the wave heights, and hence the nonlinear effects, are the greatest.

The agreement between the experimental results and the finite element model is also good for frequencies greater than that corresponding to the first resonant mode. Of the records shown, the one corresponding to $\omega L /\left(g h_{0}\right)^{\frac{1}{2}}=1.26$ appears to be the slowest to reach steady-state. In fact, it does not reach steady-state within the eight 


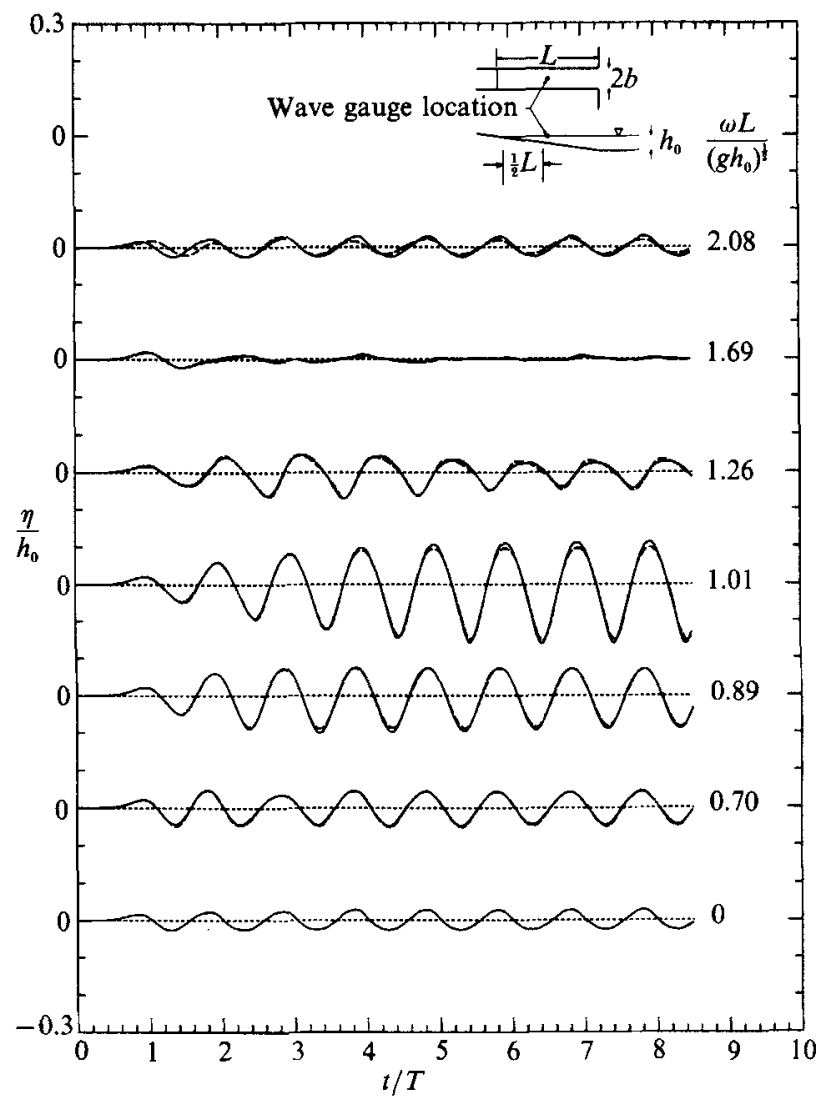

Figure 7. Wave records measured at the centre of the harbour. Comparison of laboratory experiments with the finite element model for different values of $\omega L /\left(g h_{0}\right)^{\frac{1}{2}}$. — - experiment; --- , finite element model.

periods of excitation shown, and the maximum harbour response appears to occur during the transient portion of the motion. These characteristics were present to some degree in most of the experiments for frequencies just above resonance, i.e. for harbour lengths greater than that corresponding to the first resonant mode, and it illustrates the importance of using a transient model to determine the maximum harbour response. The harbour response for $\omega L /\left(g h_{0}\right)^{\frac{1}{2}}=1.69$ is small because a node of the steady-state response coincides with the measurement location. The agreement for $\omega L /\left(g h_{0}\right)^{\frac{1}{2}}=2.08$ is still fairly good, although there is a small phase shift between the two records which can be seen during the first period of motion. (The two records were aligned to maximize the agreement of the established motion after the arrival of the incident wave system.)

It appears that the use of an entrance loss coefficient $f$ slightly less than the value of 0.8 actually used would provide better agreement between the present experiments and the finite-element model because it would increase the amplitude of the finite element results near resonance. The agreement in the response away from resonance would remain good because it is relatively insensitive to the value of the entrance loss coefficient. However, any effort spent making small adjustments in the entrance loss coefficient to obtain even better agreement is of doubtful value since it is not reasonable to consider the coefficient $f$ to be a universal constant; it is a weak 


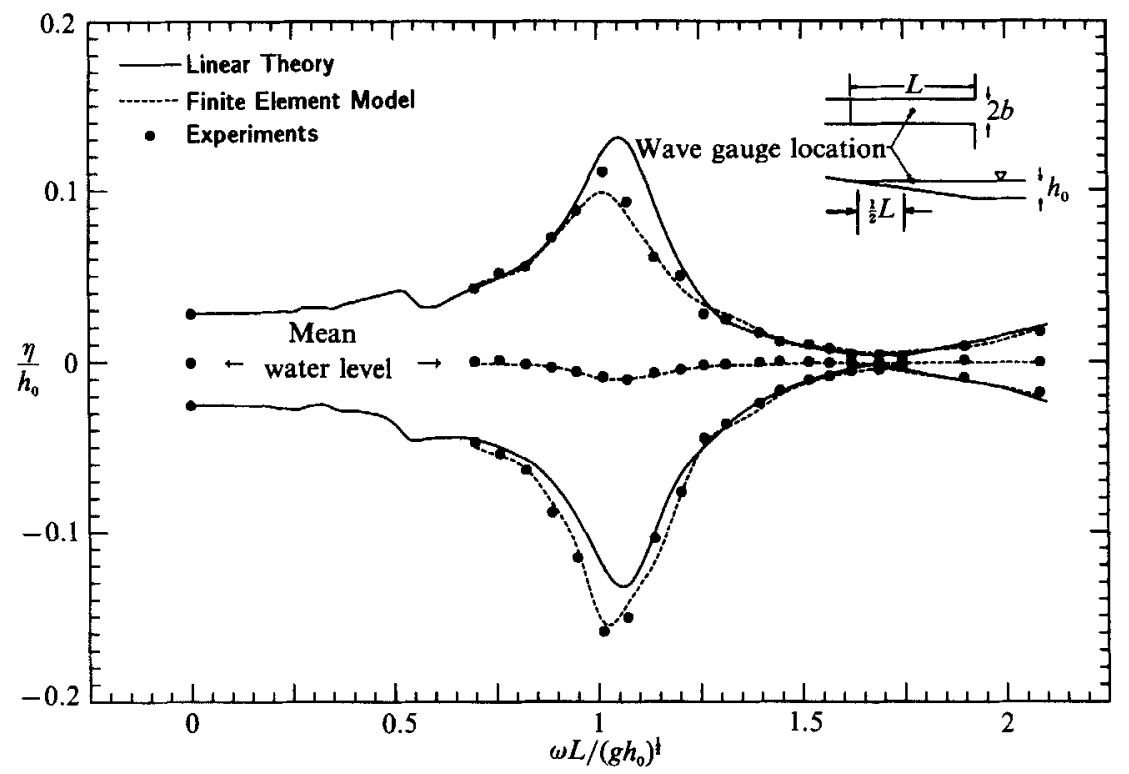

FIGURE 8. Variation of the wave extrema and mean water level with $\omega L /\left(g h_{0}\right)^{\frac{1}{2}}$, at the centre of the harbour. - linear theory; ---, finite element model; O experiments.

function of many variables as discussed by Lepelletier (1980). For numerical studies it must even be considered a function of the grid resolution at the harbour entrance (Zelt 1986). Grid refinement tests showed that the maximum harbour response at resonance increased with greater grid resolution at the entrance. Due to the finite grid spacing used to estimate derivatives and to interpolate variables, the harbour entrance is effectively spread over a wider area when a lower resolution grid is used, increasing the radiation damping and reducing the response at resonance.

The results of the experiments are summarized by the response curve presented in figure 8 , where the data are compared with the results of the finite-element model (dashed lines). Three different quantities are shown as a function of the parameter $\omega L /\left(g h_{0}\right)^{\frac{1}{2}}$ : the steady-state wave-height maxima, the wave-height minima, and the time averaged mean water level. The harbour response computed using linear theory (solid lines) is also presented in figure 8 . These results were obtained by matching at the harbour entrance the complex amplitudes of the corresponding Fourier components of three periodic solutions : the standing incident-reflected wave outside the harbour, the radiated wave, and the standing wave within the harbour itself. The radiated wave and the standing wave within the harbour were obtained theoretically, neglecting nonlinear and dispersive effects, and the incident-reflected wave was defined experimentally by the steady-state wave record with $\omega L /\left(g h_{0}\right)^{\frac{1}{2}}=0$ in figure 7 . Two matching conditions were imposed to ensure conservation of mass and to impose a head loss to account for entrance dissipation. Instead of the nonlinear expression $\Delta \eta=(f / 2 g) U|U|$ used in the numerical model, the equivalent linear loss: $\Delta \eta=r U$ was used (Rogers \& Mei 1978), where $r=4 f U_{\max } / 3 \pi g$ and $U_{\max }$ is the maximum horizontal velocity through the gap. This expression can be applied to period flows which are not sinusoidal, e.g. the experiments discussed in this paper, although its accuracy diminishes as a larger fraction of the energy in the incident wave is found in higher harmonics. For flows which are not sinusoidal the maximum 
velocity, $U_{\max }$, is a function of all the Fourier components. This introduces a weak nonlinear coupling of the Fourier modes so that a simple, but efficient, numerical technique must be used to compute the Fourier amplitudes (Zelt 1986).

In the discussion of figure 7 it was noted that for $\omega L /\left(g h_{0}\right)^{\frac{1}{2}}>1$ a definite steadystate response was not always reached within the first eight oscillations. For these cases, the response for both the experimental results and the finite element model results displayed in figure 8 was arbitrarily defined using the average of the maxima and the average of the minima for the seventh and eighth oscillations of the wave. Similarly, the mean water level was defined by the mean value of the wave record for the seventh and eighth periods of oscillation. This limitation, due to the size of the experimental wave basin, is not critical since the same quantities are compared for both the experiments and the finite element model.

The finite element model agrees better with the experimental results than does the linear theory in figure 8, and the depression of the mean water level near $\omega L /\left(g h_{0}\right)^{\frac{1}{2}}$ $=1$ is reproduced well. However, the linear-theory predictions are reasonable and useful for a first estimate of the steady-state solution, although the technique is difficult to apply to more general harbour geometries where numerical integration is necessary to obtain the wave within the harbour corresponding to the boundary condition of perfect reflection from the shore line. The linear theory predicts that the mean water level coincides everywhere with the still water level $\eta / h_{0}=0$ since there is no mechanism to transfer energy into the zero-frequency harmonic component. Partly for that reason, the linear theory overpredicts the maxima and underpredicts the minima in the harbour response. The wave-height extrema are predicted well by the finite element model, although it underpredicts both the peaks and the troughs slightly near resonance. As discussed previously, this could be improved by decreasing the entrance loss coefficient $f$ a small amount. The small peaks in the linear reponse curves in figure 8 near $\omega L /\left(g h_{0}\right)^{\frac{1}{2}}=0.5$ are due to the response of the second harmonic component in the incident wave to the first resonant mode.

\section{Summary and conclusions}

Long-wave equations in the Lagrangian description have been derived which account for weakly nonlinear and dispersive processes for wave propagation in two horizontal directions. A finite element formulation for these equations was developed to treat regions of arbitrary shape with vertical or sloping boundaries to determine the response of such regions to transient long waves. To confirm the boundary condition for the sloping boundaries of the harbour and the treatment of nonhydrostatic effects, the results of the model were compared to two cases where the exact solutions are known: one that emphasizes the nonlinear aspects of the run-up of non-breaking waves on a sloping boundary and one which emphasizes dispersive effects due to non-hydrostatic processes. The model is able to reproduce the nonlinear run-up effects well, even for the limiting case where the tangent to the water surface approaches vertical. The treatment of non-hydrostatic vertical fluid accelerations by the model greatly extends the range of wavelengths over which the phase speed is computed accurately (as defined by linear dispersive theory). The finite element model was used also to study the first-mode response of a long narrow harbour where the depth decreased linearly from the entrance to the shore line, and the results were compared to those from laboratory experiments. Overall agreement between the experiments and the finite element model was very good for both the transient and the steady-state response. Indeed the agreement was better than that between the 
experiments and a linear non-dispersive steady-state harbour response theory. The water surface time histories, recorded along the centreline of the harbour midway between the entrance and the quiescent shore line, were asymmetric, with larger troughs than crests. This nonlinear effect was most apparent near the first resonant mode where the harbour response was the greatest. As a result of this asymmetry, the mean water level at the point of measurement was negative. These features were reproduced accurately by the finite element model and were seen in the investigation of the reflection characteristics of a nonlinear wave from a sloping boundary.

This research was conducted at the California Institute of Technology and supported by the National Science Foundation Grant Nos. CEE79-12434, CEE8115457, and CEE84-10087. J.A.Z. would like to thank John Hall for many helpful suggestions, and the Australian Marine Sciences and Technologies Grant Scheme (Grant No. 84-1894) for providing funding while writing part of this manuscript.

\section{Appendix. Finite element formulation}

The finite element equations, based on (7), can be expressed in matrix form as $\boldsymbol{M} \ddot{d}+\boldsymbol{C} \dot{d}=f$, where the mass matrix $\boldsymbol{M}$, the dissipation matrix $\boldsymbol{C}$, and the forcing vector $f$ can be assembled from the element matrices $M^{\mathrm{e}}, \boldsymbol{C}^{\mathrm{e}}$, and element vectors $\boldsymbol{f}^{\mathrm{e}}$, using standard finite element assembly procedures. Central differencing was employed for the temporal integration because of its simplicity. For an element with $n$ nodes, the element matrix $\boldsymbol{M}^{\mathrm{e}}$ can be expressed as a partitioned matrix of the form :

$$
\boldsymbol{M}^{\mathrm{e}}=\left(\begin{array}{c|c|c|c}
m_{11} & m_{12} & \cdots & m_{1 n} \\
\hline m_{21} & m_{22} & \cdots & m_{2 n} \\
\hline \vdots & \vdots & \ddots & \vdots \\
\hline m_{n 1} & m_{n 2} & \cdots & m_{n n}
\end{array}\right)
$$

where the $m_{i j}$ are the $2 \times 2$ matrices:

$$
\begin{aligned}
& m_{i j}=\iint_{\Omega^{\mathrm{e}}}(1+\alpha \boldsymbol{\nabla} \cdot \boldsymbol{x})\left[\begin{array}{cc}
N_{i} N_{j} & 0 \\
0 & N_{i} N_{j}
\end{array}\right] \mathrm{d} a \mathrm{~d} b \\
& +\beta \iint_{\Omega^{\mathrm{e}}}\left\{\left[\begin{array}{cc}
\frac{\partial N_{i}}{3} h^{2}\left[\frac{\partial N_{j}}{\partial a}\right. & \frac{\partial N_{i}}{\partial a} \frac{\partial N_{j}}{\partial b} \\
\frac{\partial N_{i}}{\partial b} \frac{\partial N_{j}}{\partial a} & \frac{\partial N_{i}}{\partial b} \frac{\partial N_{j}}{\partial b}
\end{array}\right]+\frac{1}{6} h N_{i}\left[\begin{array}{ll}
\frac{\partial h}{\partial a} \frac{\partial N_{j}}{\partial a} & \frac{\partial h}{\partial a} \frac{\partial N_{j}}{\partial b} \\
\frac{\partial h}{\partial b} \frac{\partial N_{j}}{\partial a} & \frac{\partial h}{\partial b} \frac{\partial N_{j}}{\partial b}
\end{array}\right]\right\} \mathrm{d} a \mathrm{~d} b
\end{aligned}
$$

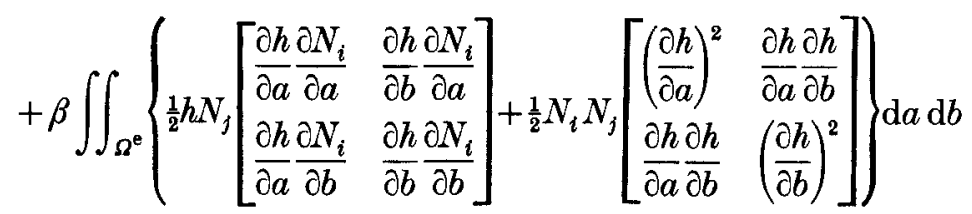

$$
\begin{aligned}
& -\beta \int_{\partial \Omega^{\mathrm{e}}}\left\{\frac{1}{3} h^{2} N_{i}\left[\begin{array}{cc}
n_{a} \frac{\partial N_{j}}{\partial a} & n_{a} \frac{\partial N_{j}}{\partial b} \\
n_{b} \frac{\partial N_{j}}{\partial a} & n_{b} \frac{\partial N_{j}}{\partial b}
\end{array}\right]+\frac{h}{2} N_{i} N_{j}\left[\begin{array}{cc}
n_{a} \frac{\partial h}{\partial a} & n_{a} \frac{\partial h}{\partial b} \\
n_{b} \frac{\partial h}{\partial a} & n_{b} \frac{\partial h}{\partial b}
\end{array}\right]\right\} \mathrm{d} s
\end{aligned}
$$


The terms $n_{a}$ and $n_{b}$ are the $a$ and $b$ components of the outward normal unit vector along the boundary of the element domain $\Omega^{\mathrm{e}} . N_{i}$ is the shape function for the element corresponding to local node number $i$. Note that if dispersion is neglected $(\beta=0), M$ is symmetric but not otherwise.

The dissipation matrix, $C$, can be expressed as a partitioned matrix similar to $\boldsymbol{M}^{\mathrm{e}}$ in (A 1), where in this case the $c_{i j}$ are the $2 \times 2$ matrices:

$$
c_{i j}=\int_{\partial \Omega^{\mathrm{e}}} N_{i} N_{j}\left[\begin{array}{ll}
n_{a} \cos \theta & n_{a} \sin \theta \\
n_{b} \cos \theta & n_{b} \sin \theta
\end{array}\right] \mathrm{d} s,
$$

and the integral is only evaluated along the radiation boundary where $(9)$ is imposed.

The element forcing vector $f^{\mathrm{e}}$ can be expressed as

$$
f^{\mathrm{e}}=\left(f_{1}^{\mathrm{T}} f_{2}^{\mathrm{T}} \ldots f_{n}^{\mathrm{T}}\right)^{\mathrm{T}}
$$

where the $f_{i}$ are the $2 \times 1$ vectors:

$$
\begin{aligned}
& f_{i}=-\iint_{\Omega^{\mathrm{e}}}\left(\left(\begin{array}{c}
1+\alpha \frac{\partial y}{\partial b} \\
-\alpha \frac{\partial x}{\partial b}
\end{array}\right) \frac{\partial N_{i}}{\partial a}+\left(\begin{array}{c}
-\alpha \frac{\partial y}{\partial a} \\
1+\alpha \frac{\partial x}{\partial a}
\end{array}\right) \frac{\partial N_{i}}{\partial b}\right. \\
& \times\left\{\boldsymbol{\nabla} h \cdot \boldsymbol{x}+\frac{1}{2} \alpha \boldsymbol{x} \cdot \boldsymbol{H} \cdot \boldsymbol{x}+h \frac{\left[\boldsymbol{\nabla} \cdot \boldsymbol{x}+\alpha \frac{\partial(x, y)}{\partial(a, b)}\right]}{1+\alpha \boldsymbol{\nabla} \cdot \boldsymbol{x}}\right\} \mathrm{d} a \mathrm{~d} b \\
& +\int_{\partial \Omega^{\mathrm{e}}} \frac{1}{2} \alpha f U|U|\left(\begin{array}{l}
n_{a} \\
n_{b}
\end{array}\right) N_{i} \mathrm{~d} s-\frac{1}{2 R_{o}} \int_{\partial \Omega^{\mathrm{e}}} \boldsymbol{x} \cdot \boldsymbol{n}_{\theta}\left(\begin{array}{c}
n_{a} \\
n_{b}
\end{array}\right) N_{i} \mathrm{~d} s \\
& -\int_{\partial \Omega^{\mathrm{e}}}\left\{\left(\begin{array}{c}
1+\alpha \frac{\partial y}{\partial b} \\
-\alpha \frac{\partial x}{\partial b}
\end{array}\right) n_{a}+\left(\begin{array}{c}
-\alpha \frac{\partial y}{\partial a} \\
1+\alpha \frac{\partial x}{\partial a}
\end{array}\right) n_{b}\right\} N_{i} \eta \mathrm{d} s
\end{aligned}
$$

Boundary conditions (8) and (11) are imposed by substituting these expressions for $\eta$ into the last boundary integral in (A 2) along the segments of the boundary where they apply. The quadratic head loss integral is evaluated along $\Gamma_{\mathbf{H M}}$, the line segment within the domain across which the head loss occurs. The boundary integral involving $R_{\mathrm{o}}$ is only evaluated along the radiation boundary.

Unlike boundary conditions (8) or (11), the boundary condition for a vertical wall, (10), does not involve specifying the water surface elevation, $\eta$, along the boundary, so it must be treated differently. Two independent degrees of freedom are associated with each node point within the fluid, corresponding to two components of displacement of the fluid particle. However, along a vertical wall there is only one degree of freedom corresponding to the component of particle displacement tangential to the wall. When the finite element equations are assembled, an orthogonal transformation is performed on the element matrices and vectors to rotate the degrees of freedom associated with nodes along a vertical wall so that one degree of freedom is tangential to the wall and the other one is perpendicular to the wall. The degrees of freedom associated with motion normal to the wall are set to zero and the equations corresponding to components of displacement parallel to the wall are assembled. In fact, instead of setting the normal components of acceleration to 
zero, these may be arbitrarily specified to model the flow induced by a moving vertical wall, including the effects of finite displacement.

\section{REFERENCES}

Bowes, A, J. 1969 Rip currents, 1, Theoretical investigations. J. Geophys. Res. 74, 23, 5467-5478. BowEN, A. J. \& INMAN, D. L. 1969 Rip currents, 2, Laboratory and field observations. J. Geophys. Res. 74, 5479-5490.

Carrier, G. F. \& Gremsspan, H. P. 1958 Water waves of finite amplitude on a sloping beach. J. Fluid Mech. 4, 97-109.

Connor, J. J. \& Brebbia, C. A. 1976 Finite Element Techniques for Fluid Flow. Butterworth.

Eagleson, P.S. \& Dean, R. G. 1966 Small amplitude wave theory. In Estuary and Coastline Hydrodynamics (ed. A. T. Ippen). McGraw-Hill.

Eckart, C. 1963 Some transformations of the hydrodynamic equations. Phys. Fluids 6, 1037-1041.

Gopalakrishnan, T. C. \& Tung, C. C. 1983 Numerical analysis of a moving boundary problem in coastal hydrodynamics. Intl J. Numer. Meth. Fluids 3, 179-200.

Goning, D. G. 1978 Tsunamis - the propagation of long waves onto a shelf. PhD thesis, W. M. Keck Laboratory of Hydraulics and Water Resources, Rep. no. KH-R-38, California Institute of Technology, Pasadena, California.

Gото, C. 1979 Nonlinear equation of long waves in the lagrangian description. Coastal Engng Japan 22, 1-9.

Goto, C. \& Sнuto, N. 1980 Run-up of tsunamis by linear and nonlinear theories. In Coastal Engineering. Proc. of the Seventeenth Coastal Engineering Conf., Sydney, Australia. ASCE, vol. 1, pp. 695-707.

GuzA, R.T. \& Davis, R. E. 1974 Excitation of edge waves by waves incident on a beach. J. Geophys. Res. 79, 1285-1291.

HeITNER, K. L. 1969 A mathematical model for calculation of the run-up of tsunamis. PhD thesis. California Institute of Technology, Pasadena, California.

HeItNer, K. L. 1970 Additional investigations on a mathematical model for calculations of the run-up of tsunamis. Earthquake Engineering Research Laboratory Rep. California Institute of Technology, Pasadena, California.

Houston, J. R. \& ButleR, H. L. 1979 A numerical model for tsunami inundation. Tech. Rep. HL-79-2. Hydraulics Laboratory, US Army Engineer Waterways Experiment Station, Vicksburg, Mississippi.

KelleR, J. B. \& KELLER, H. B. 1964 Water wave run-up on a beach. Research Report for the Office of Naval Research, Department of the Navy. Service Bureau Corporation. New York, NY.

KELLER, J. B. \& KELLER, H. B. 1965 Water wave run-up on a beach. II Research Report for the Office of Naval Research, Department of the Navy. Service Bureau Corporation. New York, NY.

LAMB, H. 1932 Hydrodynamics, 6th edn. Dover.

Lephlletier, T. G. 1980 Tsunamis-harbor oscillations induced by nonlinear transient long waves. W. M. Keck Laboratory of Hydraulics and Water Resources, Rep. no. KH-R-41, California Institute of Technology, Pasadena, California.

LoNaUET-Hragins, M. S. 1970 Longshore currents generated by obliquely incident sea waves, 1. J. Geophys. Res. 75, 6778-6789.

LyNCh, D. R. \& Gray, W. G. 1978 Finite element simulation of shallow water problems with moving boundaries. Finite Elements in Water Resources. Pentech. 2.23-2.42.

LYNCH, D. R. \& GRAY, W. G. 1980 Finite element simulation of flow in deforming regions. J. Comp. Phys. 36, 135-153.

Mei, C. C., Liv, P. \& IPPEN, A. T. 1974 Quadratic loss and scattering of long waves. J. Waterways, Harbors Coastal Engng Div. ASCE 100, WW3, August, 217-237.

MeYer, R. E. 1971 Introduction to Mathematical Fluid Dynamics. Dover. 
MrLes, J. W. 1974 Harbor seiching. Ann. Rev. Fluid Mech. 6, 17-35.

MUNGALL, J. C. H. \& REID, R. O. 1978 A radiation boundary condition for radially-spreading non-dispersive waves. Tech. Rep. 78-2-T. Texas A\&M University.

Pedersen, G. \& GJevik, B. 1983 Run-up of solitary waves. J. Fluid Mech. 135, 283-299.

Perkgrine, D. H. 1967 Long waves on a beach. J. Fluid Mech. 27, 815-827.

Raichlen, F. 1966 Estuary and Coastline Hydrodynamics (ed. A. T. Ippen). McGraw-Hill.

RAICHLEN, F. 1976 Coastal wave hydrodynamics - theory and engineering applications. W. M. Keck Laboratory of Hydraulics and Water Resources. Tech. Mem. 76-1. California Institute of Technology, Pasadena, California.

REID, R. O. \& Bodine, B. R. 1968 Numerical model for storm surges in Galveston Bay. J. Waterways Harbors Division ASCE 94, WW1, 33-57.

Rogers, S. R. \& MEI, C. C. 1978 Nonlinear resonant excitation of a long and narrow bay. J. Fluid Mech. 88, 161-180.

SIELECKI, A. \& WURTELE, M. G. 1970 The numerical integration of the nonlinear shallow-water equations with sloping boundaries. J. Comp. Phys. 6, 219-236.

Shuto, N. 1967 Run-up of long waves on a sloping beach. Coastal Engng Japan 10, 23-38.

Shuto, N. 1968 Three dimensional behavior of long waves on a sloping beach. Coastal Engng Japan 11, 53-57.

Shuto, N. 1972 Standing waves in front of a sloping dike. Coastal Engng Japan 15, 13-23.

Sнuтo, N. \& Gото, T. 1978 Numerical simulation of tsunami run-up. Coastal Engng Japan 21, 13-20.

Synolakis, C. E. 1987 The run-up of solitary waves. J. Fluid Mech. 185, 523-545.

TANakA, T., ONo, Y.\& Ishist, T. 1980 The open boundary value problems in ocean dynamics by finite elements. Finite Elements in Water Resources. Pentech. 5.47-5.63.

Tuck, E. O. \& HwaNG, L. 1972 Long wave generation on a sloping beach. J. Fluid Mech. 51, 449-461.

Ünlüata, Ü. \& MeI, C. C. 1975 Effects of entrance loss on harbor oscillations. J. Waterways Harbors Coastal Engng Div. ASCE 101, WW2, May, 161-180.

VAN DoRn, W. G. 1966 Boundary dissipation of oscillatory waves. J. Fluid Mech. 24, 769-779.

Wilson, B. W. 1972 Seiches. Adv. Hydrosci. 8, 1-94.

Yef, G.-T. \& Chou, F.-K. 1979 Moving boundary numerical surge model. J. Waterway Port Coastal Ocean Div. ASCE 105, WW3, 247-263.

ZELT, J.A. 1986 Tsunamis: the response of harbours with sloping boundaries to long wave radiation. PhD thesis. W. M. Keck Laboratory of Hydraulics and Water Resources, Rep. no. KH-R-47, California Institute of Technology, Pasadena, California, $318 \mathrm{pp}$. 